\title{
Intercâmbio de aperfeiçoamento da prática de enfermagem em terapia de acupuntura em Beijing/ China: um relato de experiência
}

RESUMO | Objetivo: Descrever e refletir sobre uma vivência internacional de aperfeiçoamento da prática de terapia da acupuntura, no âmbito das Práticas Integrativas e Complementares (PICS) na enfermagem. Método: Estudo descritivo, reflexivo, do tipo qualitativo, sob forma de relato de experiência sobre a realização de um curso de aperfeiçoamento em acupuntura em Beijing/ China. Resultados: A experiência proporcionou vivenciar diversas situações não encontradas no Brasil. As atividades totalizaram 120 horas, incluindo atividades teóricas intercaladas com atividades práticas na escola e em hospitais de Beijing que possuem alas exclusivas para as práticas de acupuntura. Conclusão: A experiência se configurou como uma oportunidade em conviver com cultura do país berço da acupuntura, agregando de forma relevante ao conhecimento teórico à técnica, pois mostrou um cenário amplo de práticas e possibilitou diversas possibilidades de tratamento não abordados no Brasil. Tal oportunidade, serviu para potencializar o conhecimento e aprimorar o cuidado de enfermagem no âmbito das PICS.

Palavras-chaves: Terapia por Acupuntura, Aprendizagem, Terapias Complementares.

\begin{abstract}
Objective: To describe and reflect about an international experience of improving acupuncture therapy practice within the scope of Integrative and Complementary Practices (PICS) in nursing. Method: Descriptive, reflective, qualitative study, in the form of an experience report on conducting an improvement course in acupuncture in Beijing/China. Results: The activities totaled 120 hours, including theoretical activities interspersed with practical activities in the school and in hospitals in Beijing that have exclusive wards for acupuncture practices. Conclusion: The experience was configured as an opportunity to live with the country's culture where acupuncture was born, adding in a relevant way to theoretical knowledge of the technique, because it showed a wide scenario of practices and allowed several possibilities of treatment not addressed in Brazil. Such opportunity served to enhance knowledge and improve nursing care within the PICS.
\end{abstract}

Keywords: Acupuncture Therapy, Learning, Complementary Therapies.

RESUMEN | Objetivo: Describir y reflexionar acerca de una vivencia internacional de mejora de la práctica de terapia de la acupuntura, en ámbito de la Prácticas Integrativas y Complementarias (PICS) en enfermería. Método: estudio descriptivo, reflexivo, del tipo cualitativo, bajo forma de informe de experiencia sobre la realización de un curso de perfeccionamiento en acupuntura en Beijing/China. Resultados: La experiencia ha proporcionado vivenciar diversas situaciones no encontradas en Brasil. Las actividades totalizaron 120 horas, incluyendo actividades teóricas intercaladas con actividades prácticas en la escuela y en hospitales de Beijing, que poseen alas exclusivas para las prácticas de acupuntura. Conclusión: la experiencia se ha configurado como una oportunidad en convivir con cultura del país de origen de la acupuntura, lo que ha agregado de manera relevante el conocimiento teórico a la técnica, pues, ha mostrado un escenario amplio de prácticas y ha permitido otras posibilidades de tratamiento no abordadas en Brasil. Tal oportunidad ha servido para potencializar el conocimiento y mejorar la atención de enfermería en el ámbito de las PICS.

Descriptores: Terapia por Acupuntura. Aprendizaje. Terapias Complementarias.

\section{Carina Suzana Pereira Corrêa}

Enfermeira. Mestranda do Programa de Pós graduação em Promoção da Saúde Universidade de Santa Cruz do Sul (UNISC).

\section{Suzane Beatriz Frantz Krug}

Enfermeira. Doutora em Serviço Social (PUC/RS). Docente do Departamento de Ciências da Saúde e do Programa de Pós graduação em Promoção da Saúde Universidade de Santa Cruz do Sul (UNISC).

Recebido em: 09/12/2019

Aprovado em: 03/03/2020
INTRODUÇÃO

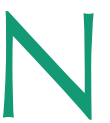
a enfermagem atual, verifica-se a importância de uma formação mais crítica e reflexiva em relação às necessidades da população. Com isso, novas práticas vêm sendo incluídas nos currículos e no dia a dia dos profissionais enfermeiros, buscando uma assistência mais integral, completa e personalizada. A atuação do profissional enfermeiro é extremamente relevante no âmbito de buscar a prevenção de morbidades dos pacientes que são atendidos por ele, implementando diversas práticas, entre elas as integrativas e complementares, com ênfase na acupuntura ${ }^{1}$.

Entre essas práticas encontram-se as Práticas Integrativas e Complementares (PICS), recursos terapêuticos que estimulam mecanismos naturais de prevenção e promoção da saúde desenvolvendo um vínculo terapêutico entre o paciente e o profissional. Ao longo dos anos, foram várias denominações para a PICS, sendo a primeira pela Organização Mundial de Saúde (OMS) que as denominou como Medicina Tradicional e Complementar ou Alternativa (MT/MCA). A denominação "Práticas Integrativas e Complementares" surge no Brasil em torno de 2006 quando foi aprovada a Política Nacio- 
nal de Práticas Integrativas e Complementares (PNPIC), e é adotada pelo Ministério da Saúde (MS) por serem conhecidas como terapias não convencionais ${ }^{2}$.

As PICS possuem diversos conceitos baseados no uso dos canais de energia como um importante comunicador dos órgãos e vísceras com o exterior e vice-versa. Cada órgão ou víscera apresenta em si, diversos sinais ou sintomas demonstrando seu desequilíbrio, permitindo fazer um diagnóstico do paciente conforme a MTC em um determinado momento da vida do ser humano ${ }^{3}$. Essas práticas são antigas e têm sua redescoberta na atualidade pelos povos do ocidente, com um crescente interesse da população e de profissionais de saúde, o que diminui o uso de alopatias ${ }^{4}$.

No âmbito das PICS, uma das mais conhecida é a terapia por acupuntura, foi desenvolvida na China há milhares de anos, sem uma definição exata do seu início, causando grande impacto na saúde da população e interesse internacional na técnica ${ }^{5}$. A ocidentalização da acupuntura trouxe a inserção de agulha como uma harmonização de energia e o fortalecimento dos órgãos e vísceras ${ }^{6}$.Abordada internacionalmente, a "acupuntura pode ser definida como terapia fisiológica coordenada pelo cérebro, que responde à estimulação dos nervos sensoriais periféricos pela inserção de agulhas por via manual ou elétrica" ${ }^{\prime \prime}$ e ainda que a técnica de inserção das agulhas estimula e aumenta a atividade das células do sistema imunológico, controlando de uma forma mais eficaz os processos inflamatórios ${ }^{5}$.

Sabe-se que o país berço da acupuntura é a China, sendo assim, as escolas que fornecem os cursos de especialização sobre essa prática estimulam o aperfeiçoamento da técnica através de oportunidades de intercâmbios, possibilitando o contato direto com o país de origem, com os locais e profissionais que praticam a técnica há muitos anos.

Com isso, o intercâmbio do ensino visa o desenvolvimento humano, ampliando horizontes culturais e possibilidades científicas e tecnológicas com a vivência internacional. Essa troca, fortalece o po- tencial de um novo aprendizado ao estudante, proporcionando um crescimento profissional na área do conhecimento e experiências ampliando seu olhar crítico para a saúde da população, possibilitando contato com outros locais de ensino, pessoas ou hábitos diversos, favorecendo a ampliação das habilidades diversas ${ }^{7}$.

A presente experiência de intercâmbio começou a ser pensada após contatar e conhecer verbalmente as experiências de outros pós-graduandos que já haviam passado por este mundo de oportunidades, percebendo-se o quanto uma mobilidade internacional pode capacitar para a vida pessoal, social e profissional. Seguindo estes conceitos, foi idealizado o estudo para servir como um estímulo aos pós-graduandos na área a buscarem o conhecimento direto na fonte de origem, sem os vieses existentes pelas traduções e entendimentos dos diversos autores estudados acerca do tema.

Sendo assim, o presente estudo tem como objetivo descrever e refletir sobre uma vivência internacional de aperfeiçoamento da prática de terapia da acupuntura, no âmbito das Práticas Integrativas e Complementares (PICS) na enfermagem.

\section{MÉTODO}

Trata-se de um estudo do tipo relato de experiência, descritivo, reflexivo, do tipo qualitativo sobre a realização de intercâmbio de aperfeiçoamento como enfermeira acupunturista do Brasil na World Federation of Acupuncture and Moxibustion Societis (WFAS) em Beijing, na China após concluir a Especialização em Acupuntura no Brasil, no ano de 2014.

Beijing ou Pequim, capital da China, é a terceira cidade mais populosa do mundo. Localizada ao norte da China, é governada sob uma administração direta com dezesseis distritos. É considerada uma cidade referência na cultura, política, ciência e economia. Suas universidades são consideradas as meIhores do país e é um centro de inovação científica e tecnológica.

A parte teórica do curso foi realizada na WFAS e teve duração de 15 dias, com aulas diárias de 8 horas e turma de 12 alunos. $\mathrm{O}$ professor foi o mesmo durante todo o período, um acupunturista chinês com mais de 20 anos de experiência demonstrada pela sua habilidade e total domínio da técnica. Ele abordou assuntos de acordo com as patologias da programação, sendo cada dia apresentada uma doença específica e o tratamento mais adequado com a técnica de acupuntura. Após a explanação teórica, era realizada a parte prática com a participação de um aluno voluntário para a demonstração da técnica pelo professor e posteriormente a prática era realizada por todos os alunos presentes na aula.

Essa vivência teve uma carga horária de 120 horas com professores nativos com uma vasta experiência na área. Considerando que a língua nativa é o mandarim, as aulas tinham tradução simultânea por um professor português, o qual era acupunturista na China há mais de 10 anos. As aulas teóricas tiveram ênfase nas doenças crônicas não-transmissíveis (DCNT) e doenças traumatológicas. Após, foram realizados estágios nos hospitais locais, aplicando as técnicas e os ensinamentos recebidos, sempre com o acompanhamento de professores supervisores, dessa vez com profissionais acupunturistas chineses que falavam espanhol.

\section{RESULTADOS}

Diante da relevância profissional e intelectual que a realização das práticas integrativas em saúde, através de intercâmbio pode proporcionar, além da oportunidade de crescimento pessoal e cultural, percebeu-se algumas dificuldades e desafios enfrentados pelos estudantes brasileiros no exterior, como por exemplo: o idioma, afetando a comunicação e dificultando a aprendizagem, pois mesmo com tradutores presentes em todas as aulas, nenhum era brasileiro, podendo comprometer o entendimento. No curso haviam em torno de 15 alunos, todos brasileiros, pois a WFAS realiza os cursos com turmas fechadas separadas por países.

A experiência no berço das PICS proporcionou um crescimento profissional e 
pessoal imenso. Após os 15 dias de aulas teóricas, tivemos cinco dias de estágio em dois hospitais da capital, com o intuito de conhecer a realidade dos serviços de saúde. As aulas teóricas tiveram como temas patologias ortopédicas e seu tratamento, sendo abordadas: hérnias de disco, dores musculares, artrites, doenças da coluna de modo geral, entre outras. Muitas técnicas receberam uma adaptação ocidental para serem utilizadas aqui, percebe-se isso nas visitas aos locais de estágio.

Foram também realizadas visitas técnicas nos serviços de saúde ao Hospitais: Beijing Tongren Hospital - uma instituição especializada em oftalmologia e otorrinolaringologia. Registros afirmam que foi inaugurado em 1886 por uma igreja metodista americana; e Beijing Hospital - hospital geral, público, especializado em geriatria, ensino e pesquisa. Construídos em 1905 por alemães; desempenha um papel importante no fornecimento de serviços médicos e de saúde a funcionários, bem como pacientes de Beijing e outras cidades da China. Essas visitas foram fundamentais para conhecer a realidade da saúde na região, possibilitando além de acompanhar e aperfeiçoar a técnica de acupuntura, de traçar um paralelo comparativo entre as diferentes realidades da China e do Brasil.

Após as 120 horas de estudo teórico-prático, houve a Certificação Diferenciada dos Estudos Avançados de Acupuntura (Advanced Studies of Acupuncture) emitida pela WFAS com o reconhecimento internacional relacionado com a World Health Organization $(\mathrm{WHO})$ e entregue pelo presidente da WFAS Professor Deng Liangye.

O intercâmbio proporcionou uma troca de conhecimento imensurável, sendo que algumas das técnicas utilizadas na China, não foram possíveis serem implementadas aqui no Brasil, ou por não ter o material disponível no país, não tendo autorização da Agência Nacional de Vigilância Sanitária (ANVISA) para a utilização, ou por se tratar de uma técnica muito antiga e um tanto quanto impactante para os ocidentais. Se muitos já são descrentes dos efeitos das técnicas habituais, não podemos prever qual seria a reação com as técnicas mais ancestrais.

\section{DISCUSSÃO}

A medicina tradicional chinesa (MTC) tem por base a integração e interação entre o ser humano e a natureza, a manutenção da saúde e a prevenção da doença, visando a harmonizar o estado de saúde geral das pessoas $^{5}$. As PICS buscam a melhoria no atendimento da população vindo ao encontro com os avanços na qualidade de vida, maior contato e aproximação com o paciente e a cultura local ${ }^{8}$.

A palavra acupuntura, foi introduzida no ocidente, no início do século XX, pelos jesuítas ao retornarem da China e provém do latim em que "acus" significa agulha e "punctura", picar'. O Conselho Nacional de Saúde (CNS) reforça que, no Brasil, a acupuntura é regularizada e fiscalizada por diversos conselhos de saúde por meio de resoluções específicas, garantindo segurança e eficácia à técnica9 ${ }^{9}$.

Literatura antiga e consagrada na área, conceitua pontos de acupuntura como sendo canais de energia, os quais se projetam na pele representando uma relação do interior com o exterior, ou seja, dos órgãos e das vísceras6 e quando as agulhas penetram nos músculos, produzem uma sensação muitas vezes não dolorosa, significando que o Qi (fluxo da energia vital) foi alcançado ${ }^{5}$.

No Brasil, criou-se a Portaria $n^{\circ}$ 971, de 03 de maio de 2006, a qual aprova a Política Nacional das Práticas Integrativas e Complementares (PNPIC) no Sistema Único de Saúde (SUS), definindo a acupuntura como uma tecnologia de intervenção em saúde que aborda o processo saúde doença no ser humano, podendo ser usada isolada ou de forma integrada com outros recursos terapêuticos que tem como objetivo a promoção e recuperação da saúde e prevenção de doenças ${ }^{11}$.

A acupuntura foi uma das primeiras a serem ofertadas pela PNPIC em 2006. Em março de 2018, o COFEN demonstra apoio à implementação das PICS no Brasil, considerando um avanço ao SUS e à profissão de enfermagem. O enfermeiro vem sendo cada vez mais importante no uso das PICS, uma vez que se fez necessárias novas abordagens na área com o intuito de uma aplicação mais sólida dos princípios do SUS pelos profissionais ${ }^{12}$.

O profissional enfermeiro, vem ganhando mais espaço nas diversas áreas de atuação da acupuntura no país, como um complemento ao tratamento, promovendo um resultado efetivo nas mais variadas patologias e nos diversos cenários da saúde atual. As terapias complementares buscam promover o bem-estar e reduzir os sintomas os quais levaram o paciente a buscar o tratamento. O enfermeiro é visto como um dos principais atores no campo das PICS como segundo maior em número de profissionais da área, ficando apenas atrás da medicina, e como um importante disseminador do conhecimento das práticas para a população em geral e aos profissionais da saúde ${ }^{12}$.

A inserção do profissional enfermeiro na acupuntura vem ocorrendo de forma gradual e crescente no Brasil, com um aumento relevante de suas atividades principalmente após o reconhecimento pelo Conselho Federal de Enfermagem (COFEN) em 1997, por meio da resolução COFEN - 197/1997, na qual a acupuntura se torna especialidade e/ou qualificação da enfermagem, sendo um avanço histórico para a profissão ${ }^{13,14}$.

A aplicação das técnicas de terapias complementares está em crescente ascensão em todo o mundo, com uma meIhora na sua aceitação e conhecimento por parte dos pacientes e dos profissionais de diversas áreas. A enfermagem, vem conquistando uma posição consolidada na realização e na apropriação das técnicas, com isso, a busca de qualificação é constante no empoderamento dos enfermeiros em relação a formas mais naturais de tratamentos ${ }^{13}$. O estímulo uso das PICS pela categoria, faz com que um olhar mais holístico do paciente se torne iminente, rompendo antigos paradigmas, bem como uma melhor compreensão, um melhor acolher e principalmente um cuidar mais completo, que é o foco da enfermagem ${ }^{1}$. 
Atualmente, estamos inseridos em um contexto científico composto por inúmeros pesquisadores dos mais diversos locais do mundo, onde o conteúdo científico é imediatamente disponibilizado para o meio acadêmico. Vivemos em sociedades globalizadas e o poder exercido pela globalização pode ser sentido em diferentes campos, principalmente no campo educacional. Uma das maiores riquezas na atualidade é o conhecimento, o qual pode ser adquirido de diversas formas, promovendo avanços tecnológicos amplos, impulsionando o movimento de internacionalização dos estudos ${ }^{7}$.

Salienta-se que a iniciativa de viajar requer aceitação, renúncias, imprevistos, ausências de entes queridos, além de adaptar as diferenças culturais, como a alimentação, hábitos e valores. Sendo assim, a capacidade do aperfeiçoamento em possibilitar aquisição de novos conhecimentos técnico-científicos, experiências significativas e enriquecedoras para os aspectos pessoais, acadêmicos e profissionais, os quais, culminaram com a construção deste relato foram imensas. $\mathrm{O}$ intercâmbio foi uma experiência singular, principalmente pelo acolhimento, admiração, qualidade de ensino, culturas, histórias e lugares magníficos para ampliar conhecimentos.

Podemos compreender o intercâmbio ou a internacionalização da educação superior como "o processo no qual se integra uma dimensão internacional e intercultural ao ensino, à pesquisa e aos serviços de uma instituição" ${ }^{\prime \prime}$ (p. 7). Sendo assim, torna-se fundamental a gestão para obter uma resposta sobre a efetividade em relação aos objetivos propostos pela instituição e/ou pelo aluno.

\section{CONSIDERAÇÕES FINAIS}

A enfermagem possui um papel fundamental na aplicação das técnicas complementares, considerando as PICS como um novo modelo de cuidado a ser implementado no ambiente de trabalho, associadas ao cuidado convencional e demais técnicas necessárias, para a promoção do empoderamento da prevenção da saúde dos pacientes e consequentemente uma diminuição dos custos do SUS.

A enfermagem está cada vez mais envolvida na busca de novos métodos de realizar o cuidado dos pacientes de uma maneira mais ampla e eficaz, com isso, a procura por crescimento profissional de qualidade torna-se cada vez mais relevante na enfermagem na busca de uma melhor qualificação profissional.

O intercâmbio, foi uma oportunidade para agregar valores, aprimorar o currículo, ampliar o conhecimento e a dedicação aos estudos na área. Proporcionou crescimento pessoal e profissional, vivência cultural, valores, vínculos e experiências significativas no contexto da formação em saúde, bem como do pesquisador e docente na área da saúde.

Nesse sentido, o pensamento crítico-reflexivo foi estimulado e desenvolvido a partir de situações diversas, de forma a buscar soluções diante de problemas e dificuldades com os recursos disponíveis.

Diante do exposto, é possível inferir que a mobilidade internacional pode representar um importante instrumento para uma melhora na qualidade dos profissionais de acupuntura no Brasil, visto que estimula o amadurecimento profissional e pessoal dos profissionais que atuam na técnica visando a valorização das práticas e da segurança ao paciente.

\section{Referências}

1 Calado R, Silva A, Oliveira D, Silva G, Silva J, Silva L, Lemos M, Santos R. Ensino das práticas integrativas e complementares na formação em enfermagem. Journal of Nursing UFPE on line [Internet]. 2019 jan [citado 2020 jan 13]; 13(1): 261267. Disponivel em: https://periodicos.ufpe.br/revistas/revistaenfermagem/article/ view/237094. doi: 10.5205/1981-8963-v13i01a237094p261-267-2019

2 Ministério da Saúde (BR). Secretaria de Atenção à Saúde. Departamento de Atenção Básica. Série B. Textos Básicos de Saúde Política Nacional de Práticas Integrativas e Complementares no SUS - PNPIC-SUS. Brasilia: Ministério da Saúde; 2006 3 Marques AMP;; Neto MRP. Das medicinas tradicionais às práticas integrativas de saúde: caracterização dos recursos humanos nas práticas alternativas de saúde adotadas no Distrito Federal [monografia]. Brasília (MG): Universidade de Brasília, 2010. Disponivel em: https://pesquisa.bvsalud.org/portal/resource/pt/biblio-878412 4 Fischborn AF, Machado J, Fagundes NC, Pereira NM. A Política das Práticas Integrativas e Complementares do SUS: o relato da implementação em uma unidade de ensino e serviço de saúde. Cinergis [Internet]. 2016 out [citado 2019 maio 27]; 17(4): 358-363. Disponível em: https://online.unisc.br/seer/index.php/cinergis/article/ view/8149. doi: 10.17058/cinergis.v1710.8149

$5 \mathrm{Ma}$ Y, Ma M, Cho ZH. Acupuntura para controle da dor: um enfoque integrado. São Paulo: Roca; 2006.

6 Yamamura Y. Acupuntura tradicional: A Arte de Inserir. São Paulo: Roca; 2001

7 Badke MR, Barbieri RL, Martorell-Poveda MA. INTERNACIONALIZAÇÃO DA ENFERMAGEM BRASILEIRA: DOUTORADO SANDUÍCHE NA REGIÃO DA CATALUNHA - ESPANHA. Texto e contexto - enfermagem. [Internet]. 2018 mar [citado 2019 nov 27]; $27(1$ ): e3620016. Disponível em: http://www.scielo.br/scielo.php?scrip$\mathrm{t}=$ sci_arttext\&pid=S0104-07072018000100700\&lng=en. doi:10.1590/0104-

\section{6}

8 Cruz PLB. As práticas terapêuticas não convencionais nos serviços de Saúde, revisão sistemática da literatura [dissertação]. São Carlos (SP): Universidade Federal de São Carlos, 2013. Disponivel em: https://repositorio.ufscar.br/handle/ufscar/6918 9 Knight J. Internationalization: management strategies and issues. International Education Magazine, Ottawa, 9(1): 6-22, 1993

10 Brasil. Ministério da Saúde. Conselho Nacional de Saúde. CNS faz recomendação sobre exercício da acupuntura. Brasília, 2012

11 Brasil. Ministério da Saúde. Portaria n ${ }^{\circ}$ 971, de 3 de maio de 2006. Aprova a Política Nacional de Práticas Integrativas e Complementares (PNPIC) no Sistema Único de Saúde. [Internet]. Diário Oficial da União, Brasília (DF), 2006 maio 03. Disponível em: http://bvsms.saude.gov.br/bvs/saudelegis/gm/2006/prt0971_03_05_2006.html 12 Mendes DS, Moraes FS, Lima GO, Silva PR, Cunha TA, Crossetti MGO, et al. Benefícios das práticas integrativas e complementares no cuidado de enfermagem. J Health NPEPS [Internet]. 2019 jan.jun.; 4(1):302-318. Disponivel em: https://pesquisa. bvsalud.org/portal/resource/pt/biblio-999705

13 Martins ES, Costa N, Holanda SM, Castro RCMB, Aquino PS, Pinheiro AKB. Enfermagem e a prática avançada da acupuntura para alívio da lombalgia gestacional. Acta paulista enfermagem [Internet]. 2019 out.; [citado 2020 jan 13]; 32(5): 477-484. Disponivel em: http://www.scielo.br/scielo.php?script=sci_arttext\&pi$d=50103-21002019000500003 \& \operatorname{lng}=e n$. doi: 10.1590/1982-0194201900067. 14 Conselho Federal de Enfermagem (COFEN). Resolução COFEN-197/1997. In: Conselho Regional de Enfermagem de São Paulo (COREn-SP). Documentos básicos de enfermagem: enfermeiros, técnicos, auxiliares. São Paulo; 2001. p. 159 - 60. 